\title{
Asymmetrical nostril axis angle in the post traumatic nose*
}

\author{
I. Smillie', A. Sil², R. Haddock³ N. Balaji
}

Department of Otolaryngology, Head \& Neck Surgery, Southern General, Glasgow, Scotland, United Kingdom

2Department of Otolaryngology, Head \& Neck Surgery, Monklands Hospital, Coatbridge, Glasgow, Scotland, United Kingdom

Department of General Medicine, Hamilton, New Zealand
Rhinology 51: 176-180, 2013

DOl:10.4193/Rhino12.143

*Received for publication:

August 28, 2012

Accepted: February 8, 2013

\section{Summary}

Background: Nasal tip reconstruction is a challenging part of septorhinoplasty in post-traumatic patients. Aesthetic appearance of the nose is dependent on adequate tip definition, projection and rotation. Our aim is to establish if caudal septal dislocation (secondary to fracture or subluxation) is a significant factor in causing asymmetry in nostril axis angle (NAA) to effect surgical intervention. In addition, to assess if there is an association between the side of dislocation and the magnitude of NAA.

Methodology: Photographic assessment of NAA was measured in a study group of 26 patients with caudal dislocation of the septum and a control group of 26 patients without caudal septal dislocation. All 52 patients were awaiting a septorhinoplasty procedure due to nasal trauma.

Results: Caudal septal dislocation causes a mean difference in NAA of $7.95^{\circ}$ compared to $1.38^{\circ}$ difference in the control group. We also found there is a greater degree of upward tip rotation on the side of the dislocation resulting in a larger NAA.

Conclusion: Caudal dislocation of the septum causes a significant asymmetry in NAA, with a greater degree of rotation on the side of the dislocation. We should be aware of this fallacy prior to surgery.

Key words: Nostril axis angle, nasolabial angle, tip rotation, septorhinoplasty, rhinoplasty

\section{Introduction}

Assessment of post traumatic nasal tip deformity requires a thorough understanding of the tip deformities including projection, definition and rotation. Reconstruction of the tip is common, yet a complex procedure. Within the NHS, reconstructive surgery has shown an increase in the last 10 years, with rhinoplasty being within the top five procedures for both male and females ${ }^{(1)}$. Despite this it is arguably the most difficult of facial plastic procedures with a revision rate as high as 12 percent ${ }^{(2)}$. The procedure is performed to correct both intrinsic and extrinsic pathology, correct the structural deformity and improve function by correcting airway obstruction. The difficulty is due to the variable anatomy, challenging deformities, difficult access and high patient expectations. Revisions often result in complex surgery to restore structure and function of the nose. Failure to achieve optimum outcome can have legal implications with 22 percent of litigation for aesthetic procedures being related to rhinoplasty ${ }^{(3)}$.

The aesthetic appearance of the nose is described in terms of definition, projection and rotation of the tip. Accurate assessment of nasal tip rotation is essential in pre- and postoperative evaluation of patients needing septorhinoplasty. The rotation can alter the appearance of the nose dramatically with desirable 
variations being dependant on sex, facial features and cultural background. Surgical planning and technique is dependent on tip rotation analysis and quantitative assessment using the nostril axis angle can be used for outcomes of rhinoplasty ${ }^{(4)}$.

In the post-traumatic nose a common potential variable in tip rotation analysis is dislocation of caudal septum into a nostril (secondary to fracture of the anterior septal cartilage or subluxation). Previous studies have shown that vertical malposition of the columella (hanging or retracted columella) may affect the position of the reference line for quantitative analysis (using nasolabial angle measurement) and therefore affect the assessment of tip rotation ${ }^{(5)}$. In the posttraumatic nose, malposition of caudal septum is more common than malposition of columella. A literature search failed to produce any studies looking at asymmetrical tip rotation in the post traumatic nose due to caudal dislocation of the septum.

The aims of our study are to establish if there is a significant asymmetry (profile views) in nostril axis angle (NAA) in the post traumatic nose in patients with severe caudal septal dislocation. Furthermore to find an association between the side of dislocation and the degree of NAA. Thus variations in tip rotation are directly related to the direction of septal dislocation.

\section{Materials and methods}

Patient study

We analysed lateral profile views of preoperative photographs taken in the Frankfurt horizontal plane (auriculo-orbital plane) of patients who were on a waiting list for a post traumatic septorhinoplasty procedure in an Otolaryngology department of a large district general hospital in Scotland. The patients were split into a study group and the control group with 26 patients in each. The study group had caudal septal dislocation into one nostril, narrowing the nostril size by 50\% when compared to the other side. This was ascertained by examination of the basal view of preoperative photos by the senior author (Figure 1). The Control group of 26 patients had equal nostril size on basal view with no caudal septal dislocation.

Tip rotation was assessed by measuring the nostril axis angle (NAA) from both sides, with differences between the two profile views assessed to establish a relationship to caudal dislocation of septum. The NAA is the angle between the nostril axis (which is the line drawn through the maximum length of the nostril at the midpoint of the nostril opening) as seen in the profile view and the vertical line drawn perpendicular to the Frankfurt horizontal plane (Figure 2). The higher the angle, reflects the obtuseness of the angle, which in turn reflects the over rotation of the tip. Conversely lesser the angle reflects under rotation of the tip. Three copies of black and white photographs of profile views of all 56 patients were taken and the nostril axis angle were individually analysed by three different observers, who were consultant / senior ENT surgeons.

\section{Statistical analysis}

Results from all three observers were collated and statistically analysed taking into account inter-observer and intraobserver variability. Normality assessed using the one sample Kolmogorov-Smirnov test. The $\mathrm{p}$ values resulting from the test indicated no significant deviation from normality and therefore, justified the use of parametric comparisons. Mean and standard deviations were calculated prior to comparison via the Student's t-test for significance. Inter-observer concordance was assessed using the intraclass correlation coefficient.

\section{Results}

The results of analyses of data related to our observations are subdivided into 2 groups, Group A representing the cohort of patients without a septal dislocation, and Group B representing the cohort of patients with a septal dislocation.

\section{Tip asymmetry}

Table 1 summarises the findings of the three observers who looked at the NAA measurements on the right lateral profile and on the left lateral profile of a patient. The difference between the NAA measurements of the 2 profiles was defined as tip asymmetry for purposes of this analysis. Tip asymmetries as noted by the individual observers were recorded for the 2 patient cohorts - with and without caudal dislocation. The observations for tip asymmetries in the 2 patient cohorts (with and without the caudal dislocation) were examined for normality using the one sample Kolmogorov-Smirnov test. The $p$ values resulting from the test indicated no significant deviation from normality and therefore, justified the use of parametric comparisons.

The mean and SD values of the tip asymmetries in degrees are given in Table 1. The mean value of tip asymmetry observed in the group with caudal dislocation was $7.95^{\circ}$ and the mean value of tip asymmetry observed in the group without caudal dislocation was $1.38^{\circ}$.

The mean asymmetries in the 2 groups were compared using the Student's t test and were repeated for all 3 observers, and the difference was found to be significant in all the 3 observations (Table 1).

\section{Inter-observer concordance}

We studied for inter-observer concordance for agreement between the mean difference of their individual measurements using the intraclass correlation coefficient and the correlation matrix is given in Table 2 . 


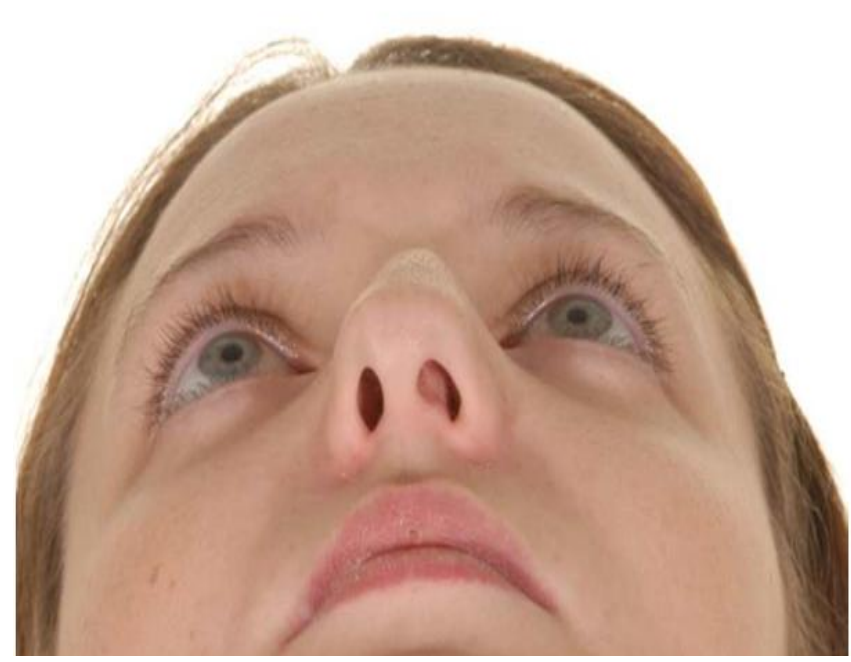

Figure 1. Basal view indicating septal displacement from columella.

Reliability statistics using intraclass coefficient and Cronbach's alpha coefficient as a measure of internal consistency showed excellent inter-observer concordance as seen in Table 2. This proves the presence of excellent inter-observer agreement.

\section{Direction of dislocation}

We have compared the mean NAA measurements on the side of the caudal dislocation of the septum and the mean NAA measurements on the side without the caudal dislocation of the septum in the cohort of patients who had the caudal dislocation. For this purpose, mean values of NAA (average of the right and
Table 1. Summary of results comparing tip asymmetries recorded in patients with and without caudal dislocation of the nasal septum.

\begin{tabular}{|c|c|c|c|}
\hline & $\begin{array}{l}\text { Group A } \\
\text { with caudal } \\
\text { dislocation } \\
\text { of septum - } \\
\text { Mean (SD) }\end{array}$ & $\begin{array}{l}\text { Group B } \\
\text { without caudal } \\
\text { dislocation of } \\
\text { septum . } \\
\text { Mean (SD) }\end{array}$ & $\begin{array}{l}\text { Result of } \mathbf{t} \text { test } \\
\text { - } \mathbf{t} \text { statistic } \\
\text { (p value) }\end{array}$ \\
\hline Observer 1 & 8.077 & 1.154 & $\begin{array}{c}6.355 \\
(p<0.001)\end{array}$ \\
\hline Observer 2 & 7.96 & 1.269 & $\begin{array}{c}6.086 \\
(p<0.001)\end{array}$ \\
\hline Observer 3 & 7.8 & 1.731 & $\begin{array}{c}5.033 \\
(p<0.001)\end{array}$ \\
\hline Mean values & 7.95 & 1.384 & \\
\hline
\end{tabular}

left sided measurements) were calculated and recorded for the 3 observers. These values were then subjected to a one sample Kolmogorov Smirnov test to assess normality and this showed no significant deviation from normality in all three observations (Table 3). The mean values of NAA observed on the dislocation side and the non dislocated side were compared using the paired sample t test. The difference reached statistical significance as shown in Table 3.

\section{Statistical Inference}

Table 3 shows that the mean NAA values were higher in the side of the dislocation compared to the opposite side. This difference reached statistical significance, leading to the rejection of the null hypothesis of no difference in the NAA sample measurements on either side of the caudal dislocation.
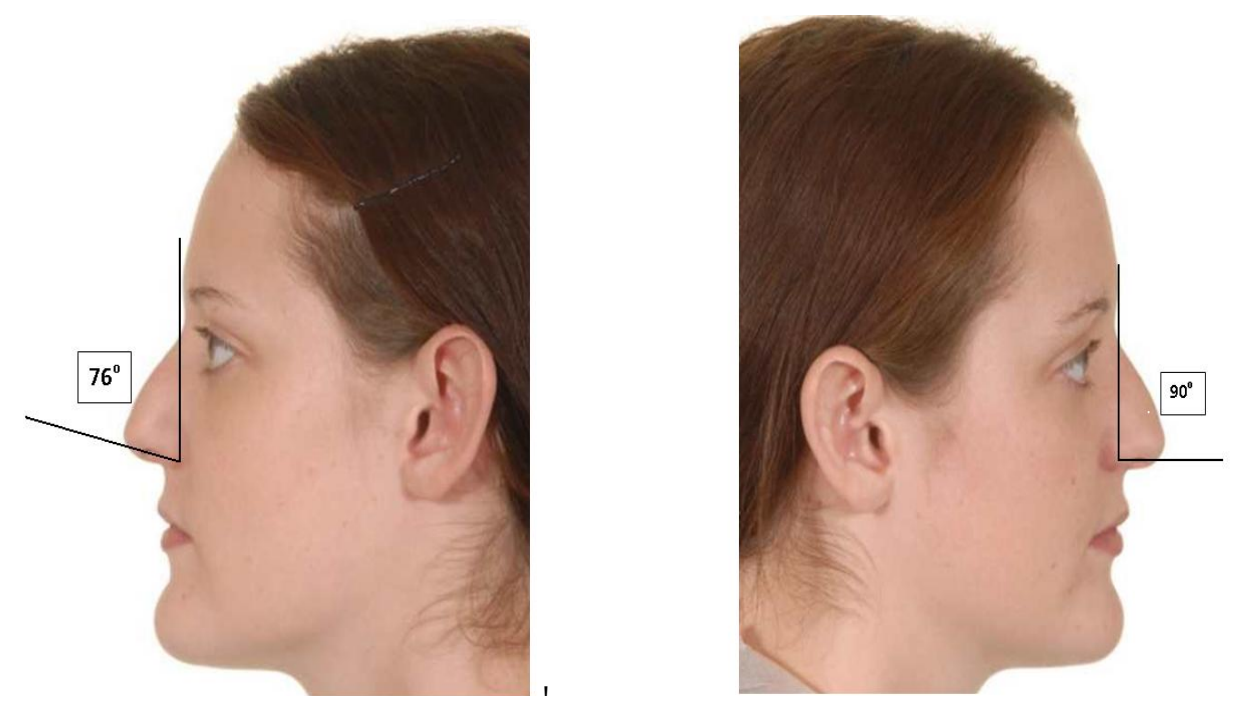

Figure 2. A) lateral view showing greater rotation of tip on side of dislocation. B) Lateral view showing reduced rotation of tip. 
Table 2. Reliability statistics and correlation matrix between the mean values of NAA measurements of both sides between 3 observers: Mean (of NAA values of right and left side) and SD.

\begin{tabular}{|c|c|c|c|c|c|c|}
\hline & & & & \multicolumn{3}{|c|}{ Correlation matrix } \\
\hline & Mean & SD & $\mathbf{N}$ & $\begin{array}{c}\text { Mean NAA } \\
\text { observer1 }\end{array}$ & $\begin{array}{l}\text { Mean NAA } \\
\text { observer } 2\end{array}$ & $\begin{array}{l}\text { Mean NAA } \\
\text { observer } 3\end{array}$ \\
\hline $\begin{array}{l}\text { mean of right } \\
\text { and left NAA } \\
\text { measurements of } \\
\text { observer } 1\end{array}$ & 93.615 & 9.273 & 52 & 1.000 & 0.996 & 0.999 \\
\hline $\begin{array}{l}\text { mean of right } \\
\text { and left NAA } \\
\text { measurements of } \\
\text { observer } 2\end{array}$ & 93.807 & 9.379 & 52 & 0.996 & 1.000 & 0.995 \\
\hline $\begin{array}{l}\text { mean of right } \\
\text { and left NAA } \\
\text { measurements of } \\
\text { observer } 3\end{array}$ & 93.528 & 9.239 & 52 & 0.999 & 0.995 & 1.000 \\
\hline
\end{tabular}

Crohnbach's alpha $=0.99$. Intraclass correlation coefficient $(\mathrm{ICC})=0.997(95 \% \mathrm{Cl} 0.995-0.998) \mathrm{p}<0.001$

Table 3. Comparison of mean values of NAA between the side of the caudal dislocation and the side opposite to the dislocation in the cohort of patients with caudal dislocation (Group B).

\begin{tabular}{|c|c|c|c|}
\hline & $\begin{array}{c}\text { Group A - } \\
\text { Mean (SD) } \\
\text { values of NAA } \\
\text { on the caudal } \\
\text { dislocation } \\
\text { side }\end{array}$ & $\begin{array}{c}\text { Group B } \\
\text {-Mean( SD) } \\
\text { values of NAA } \\
\text { opposite to } \\
\text { the side of } \\
\text { caudal } \\
\text { dislocation }\end{array}$ & $\begin{array}{c}\text { Result of } \\
\text { paired sample } \\
\text { t test } \\
\text { - T statistic } \\
\text { (p value) }\end{array}$ \\
\hline Observer 1 & $93.81(12.71)$ & 90.04 (11.61) & $\begin{array}{c}2.1 \\
(p=0.05)\end{array}$ \\
\hline Observer 2 & $94.0(12.68)$ & $89.9(11.61)$ & $\begin{array}{c}2.3 \\
(p=0.03)\end{array}$ \\
\hline Observer 3 & $93.73(12.69)$ & 90.04 (11.58) & $\begin{array}{c}2.0 \\
(p=0.05)\end{array}$ \\
\hline Mean values & 93.85 & 89.99 & \\
\hline
\end{tabular}

The results of the $t$ tests used to compare mean values of NLA measurements in patient cohorts with and without caudal dislocation of the septum were significant . Therefore this provided strong evidence to reject the null hypothesis of no difference between the tip asymmetries (as defined by difference between the NAA measurements between the right and left side) in both these groups.

The results of the reliability statistics show excellent interobserver agreement and internal consistency which validates our observations and results.

\section{Discussion}

A successful rhinoplasty involves creating symmetry and harmony with the other facial structures. Accurate pre- and post-operative assessment of the nose is essential. This enables the surgeon to establish the patient's expectations from an operation and decide on surgical technique. The lateral profile view of the nose is influenced by length, tip projection and rotation. Nasal tip rotation is the position of the tip in relation to the facial plane, which can be assessed using the nostril axis angle (NAA). Our postulation was that there is a difference between NAA as measured from both sides in patients with caudal septal dislocation. This factor should be taken into account while assessing tip rotation for both septoplasty and septorhinoplasty procedures.

Variables such as tip projection are consistently measurable, but tip rotation is often inconsistent. There are numerous methods described to measure tip rotation prior to septorhinoplasty. Historically nasolablial angle (NLA) is most commonly used for assessing tip rotation. The NLA is measured as the angle between the columella of the nose and the upper lip. The aesthetically attractive range for tip rotation is described as being an angle between $90-105^{\circ}$ in men and $105-120^{\circ}$ in females ${ }^{(6)}$. These are only a reference and must be used in the context of patient height, facial features and ethnicity ${ }^{(7)}$. However, variations in anatomical landmarks including upper lip, soft tissue columella and facial expression can make this measurement inconsistent. In addition, a large nasal spine, a tension nose deformity or a highly projected tip may cause a larger NLA. In contrast, a hypoplastic midface may cause a more acute NLA ${ }^{(8)}$. 
angle between the nostril axis line and the vertical perpendicular drawn to the Frankfurt horizontal plane. Nostril axis line is a line drawn along the longest nostril axis in the profile view. The main advantage of this method is that it is not subjected to variability due to columella or upper lip position. However a potential weakness of this measure is an inconsistency of the nostril to be in the same axis as the tip which may affect comparison of tip rotation between different patients. However, we have used this method to assess the difference between ipsilateral and contralateral side in relation to caudal septal dislocation on the same patient, hence eliminating the inconsistency.

The results of our study has shown that caudal dislocation of the septum causes significant differences in nostril axis angle as measured between both sides. The noted mean difference of 7.95 degrees in the study group can alter the aesthetic appearance significantly when seen from either side and should be taken into consideration prior to septorhinoplasty. In comparison the mean difference of 1.38 degrees noted in the control group who were also awaiting septorhinoplasty following nasal trauma indicates that this rotational asymmetry can be contributed to the caudal dislocation of the septum. Both groups did have statistically significant differences between sides but the large standardized mean difference in group $B$ indicates the importance of caudal dislocation of the septum on NAA. Furthermore, this indicates that it is not possible to put a direct measurement on what is a clinically significant asymmetry in rotation. Instead, it is essential to know the deformities that can alter rotation and assess these prior to septorhinoplasty, with the knowledge that displacement of the caudal septum will cause notable differences in NAA. In addition, there was a correlation with direction of dislocation with more obtuse angle (over rotation) being demonstrated on the side of the dislocation.

To our knowledge there have been no studies demonstrating the effect of caudal septal dislocation on NAA. Our study emphasises that while assessing tip rotation, the NAA should be measured from right and left profile views in addition to caudal septal assessment prior to selecting techniques to alter rotation. Furthermore, patients should be made aware of this deformity and discussions on ideal rotation made prior to embarking on septorhinoplasty.

\section{Conclusion}

Septorhinoplasty remains a complex facial reconstructive procedure and accurate preoperative assessment and discussion on expectations is essential to gain the desired outcomes. Definition, projection and rotation are the key areas of tip alteration and caudal dislocation of the septum is a significant factor in causing variation in nostril axis angle on profile views. This influence of caudal septal dislocation helps in diagnosing the exact pathology of the deformity and hence the management plan.

\section{Authorship contribution}

IS: data collection, author of manuscript; AS: data collection and analysis; $\mathrm{RH}$ : data collection. NB: senior author and consultant operating surgeon.

\section{Conflict of interest}

There are no conflicts of interest.

\section{References}

1. http://www.nhs.uk/conditions/Cosmeticsurgery/Pages/Introduction.aspx

2. http://emedicine.medscape.com/ article/1292426-overview\#a0199

3. http://www.phudson.com/backgroudn/ ligation.html.

4. Sporri S, Simmen D, Briner HR, Jones $\mathrm{N}$. Objective assessment of tip projection and the nasolabial angle in rhinoplasty. Arch Facial Plast Surg, 2004, 6(5):295-298.

5. Adamson PA, Tropper GJ, McGraw BL. The hanging columella. J Otolaryngol. 1990; 19:319.
6. Ingels K, Orhan K. Measurement of preoperative and postoperative nasal tip projection and rotation. Arch Facial Plast Surg 2006; (8): 411-415.

7. Honrado C, Pearlman SJ. Surgical treatment of the nasolabial angle in balanced rhinoplasty. Arch Facial Plast Surg, 2003;5(4):338344.

8. Kim DW, Egan KK. Metrics of nasal tip rotation: a comparative analysis. Laryngoscope. 2006, 116 (6): 872-7
Mr lan G. Smillie

(MBCHb, BSC MedSci, MRCSEd,

DOHNS)

Department of Otolaryngology

Head \& Neck Surgery

Gartnavel General Hospital

1053 Great Western Road

Glasgow, G120YN

Scotland, United Kingdom

E-mail: iansmillie@doctors.org.uk 